\title{
Diagnóstico y análisis de los factores que influyen en la vulnerabilidad de las fuentes de abastecimiento de agua potable a la Ciudad de México, México
}

\author{
Oscar Escolero, Stefanie Kralisch, Sandra E. Martínez, María Perevochtchikova
}

\begin{abstract}
Oscar Escolero
escolero@geologia.unam.mx

Departamento de Geología Regional, Instituto de Geología, Universidad Nacional Autónoma de México, Ciudad Universitaria, 04510,

Ciudad de México, México.
\end{abstract}

\section{Stefanie Kralisch}

Department of Applied Geosciences (AGW), Karlsruhe Institute of Technology (KIT),

Kaiserstr. 12, 76131, Karlsruhe, Germany.

\section{Sandra E. Martínez}

Departamento de Geología Regional, Instituto de Geología, Universidad Nacional Autónoma de México, Ciudad Universitaria, 04510,

Ciudad de México, México.

\section{María Perevochtchikova}

Centro de estudios ambientales, El Colegio de México AC, Camino al Ajusco $\mathrm{N}^{\circ}$ 20, Pedregal de Sta. Teresa 10740, Ciudad de México, México.

BOL. SOC. GEOL. MEX. 2016

VOL. 68 NO. 3

P. $409-427$

Manuscrito recibido: Agosto 4, 2014 Manuscrito corregido recibido: Febrero 3, 2015. Manuscrito aceptado: Abril 27, 2015.

\section{RESUMEN}

El aumento de la densidad de población y la dinámica de la expansión urbana, con una marcada tendencia al crecimiento de las zonas urbanas y disminución de la población rural, hacen a las megaciudades focos de vulnerabilidad y de alta complejidad en la tarea de prestar los servicios urbanos básicos. En el caso de la Ciudad de México, la creciente demanda de agua ha impactado de manera negativa y creciente en el balance de las cuencas y acuíferos locales y vecinos que sirven de fuentes de abastecimiento a la ciudad, generando daños económicos y ambientales que ya se muestran dramáticos. Este trabajo se enfoca en realizar un diagnóstico de las fuentes de abastecimiento de agua potable que abastecen a la mega ciudad de México y analizar los factores que inciden en su vulnerabilidad; para ello se analizan tanto factores que están directamente relacionados con la infraestructura hidráulica, los aspectos jurídico-administrativos y sociales, como otros que son de relevante importancia para la sostenibilidad de las fuentes.

Se determinó que los factores que más influyen son los relacionados con la disponibilidad presente y futura del agua, los conflictos sociales y políticos, los hundimientos del terreno por extracción intensiva de agua subterránea, la transferencia intersectorial del agua, y el deterioro ambiental de las áreas de captación de agua superficial y de recarga de los acuíferos. Se estableció que la alta vulnerabilidad por la degradación ambiental que presentan las fuentes de abastecimiento de origen superficial obliga a reevaluar los proyectos de importación de agua y que la protección de las partes altas de las cuencas debe ser considerada como uno de los temas prioritarios para la protección ambiental y civil, el cuidado de las zonas de recarga y la conservación del clima local.

Palabras clave: vulnerabilidad, abastecimiento del agua, gestión del agua, infraestructura para el agua, degradación ambiental.

\section{ABSTRACT}

The increase in population density and $d y$ namics of urban expansion, with a sharp upward trend in urban areas and declining rural population, make megacities vulnerable with a high degree of complexity in the task of providing basic urban services. In the case of Mexico City, the increase in the demand for water has negatively and increasingly impacted the balance of watersheds and aquifers that serve as local and neighboring sources of water supply, causing dramatic economic and environmental damage. This work focuses on the examination of the factors that influence the vulnerability of drinking water sources in Mexico City; we analyzed factors that are directly related to hydraulic infrastructure, legal-administrative and social aspects, as well as other issues of considerable importance for the sustainability of sources.

It was determined that the most important factors are related to the present and future availability of water, social and political conflicts, land subsidence as a result of intensive groundwater extraction, intersectoral transfer of water, and environmental degradation in areas of surface water collection and groundwater recharge. It was established that high vulnerability of surface water supply sources due to environmental degradation brings pressure to reassess water importation projects and to protect the upper parts of the basins, which should be considered as priority issues for environmental and civil protection, care of recharge areas and conservation of the local climate.

Keywords: vulnerability, water supply, water management, water infrastructure, environmental degradation. 


\section{Introducción}

El aumento de la densidad de población y la dinámica de la expansión urbana, con una marcada tendencia al crecimiento de las zonas urbanas y disminución de la población rural, hacen a las megaciudades focos de vulnerabilidad y de alta complejidad en la tarea de prestar los servicios urbanos básicos (Martínez et al., 201 1; Kraas, 2008; Mitchell, 2006).

El concepto de vulnerabilidad enfocado en los recursos hídricos ha sido aplicado desde diferentes perspectivas. Pinto et al. (2010) analizaron la vulnerabilidad de redes de tuberías de agua para identificar posibles escenarios de falla de la infraestructura; Sena et al. (2012) evaluaron la vulnerabilidad de ríos en la Cuenca del Amazonas ante eventos climáticos extremos, considerando aspectos geo-ecológicos y socio-ambientales; Wilhelmi y Morss (2013) evaluaron la vulnerabilidad ante precipitaciones extraordinarias en Fort Colins, Colorado, considerando los aspectos sociales; Pandey et al. (2010) analizaron la vulnerabilidad a sequías en la cuenca Sonar en India, considerando aspectos como la topografía, uso del suelo, demanda de agua, disponibilidad de agua y registros de precipitación; Hamouda et al. (2009) analizaron la vulnerabilidad en la cuenca del Río Nilo con base en 31 indicadores que consideran aspectos hidro-fisicos y socio-económicos; Chia-Ling y Chung-Hsu (2010) analizaron la vulnerabilidad en una cuenca de Taiwán considerando factores meteorológicos y geográficos. Sin embargo, definir criterios para cuantificar la vulnerabilidad de las fuentes de abastecimiento de agua potable no es tarea sencilla debido a la falta de consenso en el verdadero significado del término, la complejidad de los sistemas analizados y al hecho de que la vulnerabilidad no es frecuentemente un fenómeno observable (Downing et al., 2001; Luers et al., 2003; Pritchett et al., 2000). La dificultad puede aumentar cuando se evalúa un fenómeno gradual y continuo tal como el impacto del cambio climático (Olmos, 2001) o la degradación de ecosistemas. A pesar de la complejidad, se han propuesto métodos de medición cuantitativos y semi-cuantitativos utilizando, en gran parte de los casos, un conjunto o combinación de indicadores. La utilidad de usar variables (o atributos) en las determinaciones de la vulnerabilidad radica en que simplifican y resumen la información relevante; por un lado, hacen visible o perceptible el fenómeno de interés, por otro cuantifican, miden y comunican información relevante. El desarrollo de variables e indicadores para uso en modelación y evaluación, permite llevar a cabo análisis de vulnerabilidad que integran perspectivas sociales y ambientales (Moss et al., 2002).

En el presente trabajo se realiza un diagnóstico de las fuentes actuales de abastecimiento de agua potable a la Ciudad de México y se analizan los factores o atributos que influyen en su vulnerabilidad, considerando los factores ambientales, sociales, económicos y técnicos. Este trabajo es complementario con el trabajo presentado en Martínez et al. (2015), en donde se propone una metodología para evaluar la vulnerabilidad de las fuentes de abastecimiento de agua potable y se aplica dicha metodología al caso de la Ciudad de México ante varios escenarios de cambio climático, basándose en el trabajo realizado por Escolero et al. (2009).

\section{Antecedentes}

$\mathrm{El}$ agua ha sido un tema crítico para la sustentabilidad de la Ciudad de México (CDMX); que se ubica en la región política-administrativa denominada Distrito Federal, el cual lo conforman a su vez 16 delegaciones políticas. La creciente demanda de agua ha impactado de manera negativa y creciente en el balance de las cuencas y acuíferos locales y vecinos que sirven de fuentes de abastecimiento de agua potable a la CDMX, generando daños económicos y ambientales que ya se muestran dramáticos. Hay numerosos autores que han discutido los problemas de agua de la CDMX desde diferentes perspectivas (Birkle et al., 1998; Tortajada, 2006; Carrera-Hernández y Gaskin, 2009; González-Reynosa et al., 2010; Mendoza et al., 2008; El Banco Mundial, 2013). Las tradi- 
cionales prácticas de importación de agua para satisfacer la demanda urbana aunada al déficit regional, ha llevado a crecientes conflictos sociales y políticos en torno a la distribución y gestión del recurso agua. Los problemas de abastecimiento a la CDMX van más allá de sus fuentes e incluyen un complejo sistema de captación y conducción que enfrenta fuertes limitaciones. El envejecimiento de la infraestructura, los costos de operación, la falta de inversión en mantenimiento y rehabilitación, así como el deterioro de las fuentes de agua en cantidad y calidad, lo que ha llevado al sistema de abastecimiento al límite de la operatividad fisica y económica. Esto coincide con lo que encontró Hamouda et al. (2009) en la Cuenca del Nilo, donde observó que los proveedores de los servicios de agua potable se enfrentan a la falta de recursos financieros y la capacidad administrativa para el mantenimiento de la infraestructura por el envejecimiento y la creciente necesidad de desarrollar nuevas fuentes de abastecimiento, con el fin de responder al reto de la creciente demanda de agua; y considerando que en muchas regiones, el crecimiento urbano implica el uso intensivo y la contaminación de los recursos hídricos en las zonas urbanas y su periferia, seguido por el desarrollo de importaciones de agua desde largas distancias (Morris et al., 2003; Martínez et al., 2009).

\section{Objetivo}

El presente trabajo se enfoca en realizar un diagnóstico de las fuentes de abastecimiento a la Ciudad de México, en las condiciones actuales, y se analizan los factores que inciden en su vulnerabilidad. El término "vulnerabilidad" dentro del contexto de este trabajo se refiere a la propensión de que disminuya la disponibilidad de agua potable entregada a la CDMX por parte de sus fuentes de abastecimiento. Se consideran tanto factores que están directamente relacionados con la infraestructura hidráulica, los aspectos jurídico-administrativos y sociales, como otros que son de relevante importancia para la sostenibilidad de las fuentes.

\section{El abastecimiento del agua potable a la Ciudad de México}

La Ciudad de México (CDMX) se encuentra ubicada a una elevación media de $2240 \mathrm{msnm}$, en una cuenca cerrada de aproximadamente 9739 $\mathrm{km}^{2}$ de extensión territorial, rodeada de cadenas montañosas volcánicas. Con una disponibilidad de agua de $74 \mathrm{~m}^{3} /$ habitante/año, la región se enfrenta a la más baja disponibilidad de agua per cápita y la densidad de población más alta del país (CONAGUA, 2009 y 2012a).

El abastecimiento de agua potable a la CDMX incluye una compleja estructura administrativa para el manejo del agua Tabla 1, en la que interactúan organismos de diferentes niveles: federal, regional, estatal y local. La Comisión Nacional del Agua (CONAGUA) es la instancia del gobierno federal encargada de la administración del agua a nivel nacional y actúa a nivel regional a través del "Organismo de Cuenca Región XIII, Aguas del Valle de México" (OGAVM). El "Sistema de Aguas de la Ciudad de México" (SACMEX) es el organismo operador del Grobierno del Distrito Federal, encargado de abastecer de agua a la CM. Fue conformado en 2003 al fusionarse la entonces Dirección General de Construcción y Operación Hidráulica (DGCOH) y la Comisión de Aguas del Distrito Federal (CADF).

La fuente principal de agua potable a la CDMX es agua subterránea proveniente del acuífero denominado "Zona Metropolitana de la Ciudad de México" (ZMCDMX); este acuífero subyace a la CDMX (CONAGUA, 2012b). En respuesta a la creciente demanda de agua y la disminución de los niveles freáticos en los pozos localizados en la CDMX, se han construido sistemas de pozos adicionales (pozos PAI), como una solución temporal para aliviar el estrés hídrico en la década de 1970, pero que todavía continúa en operación debido a la creciente demanda de agua, y se construyeron sistemas provisionales para importar agua desde fuera de las fronteras de la ciudad, desde los es- 
tados de Hidalgo y México; entre ellos el sistema Chiconautla, ubicado a $32 \mathrm{~km}$ al norte del centro de la ciudad (CONAGUA, 2007b). Dos de los principales sistemas de importación de agua desde fuera de la CDMX son: El Sistema Lerma que se desarrolló en diferentes etapas entre los años 40's y 80's para importar agua subterránea desde la cuenca alta del río Lerma, ubicado en el vecino Estado de México. El sistema Cutzamala, construido en tres etapas a partir de 1982 es la fuente de agua superficial más importante; El agua se capta en siete presas ubicadas en la cuenca del río Cutzamala y se transporta a través de un acueducto de 127 kilómetros hacia el Estado de México y la CDMX (CONAGUA/IMTA, 2006), ver Figura 1; hay otras fuentes de abastecimiento de agua de menor importancia dentro de las fronteras de la CDMX. Un conjunto de manantiales que afloran en la Sierra del Ajusco, al sur de la CDMX. Por otro lado, los escurrimientos de agua superficial dentro de la ciudad se han convertido casi en su totalidad en drenes de aguas pluviales y desagües de alcantarillado, el único que actualmente se utiliza para el abastecimiento de agua potable es el río Magdalena, que debido al bajo caudal de cap- tación y la falta de datos, incide muy poco en el abastecimiento a la CDMX.

\section{Metodología}

\subsection{DIAGNÓSTICO DE LAS FUENTES DE ABASTECIMIENTO DE AGUA}

Se realizaron extensos diagnósticos de las condiciones actuales de las fuentes de abastecimiento, considerando el estado de la infraestructura hidráulica, el estado del medio ambiente en las zonas de influencia y la situación jurídico-administrativa de cada fuente.

En el presente trabajo se consideran todas las fuentes que abastecen de agua potable a la CDMX. Las fuentes de abastecimiento son entendidas como el conjunto formado por las áreas de captación y la infraestructura, hasta el punto de entrega a la CDMX. El área de captación corresponde a las (sub)cuencas hidrológicas en el caso de fuentes superficiales y a acuíferos en el caso de agua subterránea. Las áreas de captación se ubican en tres cuencas hidrológicas: la Cuenca del Valle de México, la Cuenca del Río Cutzamala y la Cuenca

Tabla 1. Resumen de las fuentes de abastecimiento a la Ciudad de México.

\begin{tabular}{|c|c|c|c|c|c|}
\hline Fuente & Sistema & Operador & Área de Captación & Edad & Aportación \\
\hline Pozos SACMEX & $\begin{array}{l}549 \text { pozos en la zona } \\
\text { urbana, México D.F. }\end{array}$ & SA & $\begin{array}{l}\text { Acuífero (Zona Metropolitana } \\
\text { de la Ciudad de México) }\end{array}$ & $30-60$ años & $14 \mathrm{~m}^{3} / \mathrm{s}$ \\
\hline Batería Chiconautla & 41 pozos & & Acuífero (Cuautitlán-Pachuca) & $>50$ años & $1.33 \mathrm{~m}^{3} / \mathrm{s}$ \\
\hline Sistema PAI & $\begin{array}{l}156 \text { pozos distribuidos } \\
\text { en } 7 \text { ramales o baterías }\end{array}$ & OCAVM & $\begin{array}{l}\text { Acuífero (Cuautitlán-Pachuca) y } \\
\text { Acuífero Texcoco }\end{array}$ & $\begin{array}{c}>40 \text { años con } \\
\text { reposiciones } \\
\text { posteriores }\end{array}$ & $2.83 \mathrm{~m}^{3} / \mathrm{s}$ \\
\hline Sistema Lerma & 250 pozos & SACMEX & $\begin{array}{l}\text { Acuífero Toluca y Acuífero } \\
\text { (Ixtlahuaca-Atlacomulco) }\end{array}$ & $>40$ años & $4.4 \mathrm{~m}^{3} / \mathrm{s}$ \\
\hline Sistema Cutzamala & 7 presas & OCAVM & Cuenca del Río Cutzamala & $>30$ años & $6.7-9.6 \mathrm{~m}^{3} / \mathrm{s}$ \\
\hline $\begin{array}{l}\text { Captación de } \\
\text { manantiales }\end{array}$ & 18 manantiales & SACMEX & Sierra de Ajusco, Sur de la CM & $>30$ años & $0.8 \mathrm{~m}^{3} / \mathrm{s}$ \\
\hline
\end{tabular}

SACMEX: Sistema de Aguas de la Ciudad de México, organismo del Gobierno del Distrito Federal. OCAVM: Organismo de Cuenca del Valle de México, Organismo dependiente de la Comisión Nacional del Agua, del Gobierno Federal. PAl: Programa de Acción Inmediata, programa de emergencia de perforación de pozos del Gobierno Federal. Fuente: CONAGUA, 2009. 

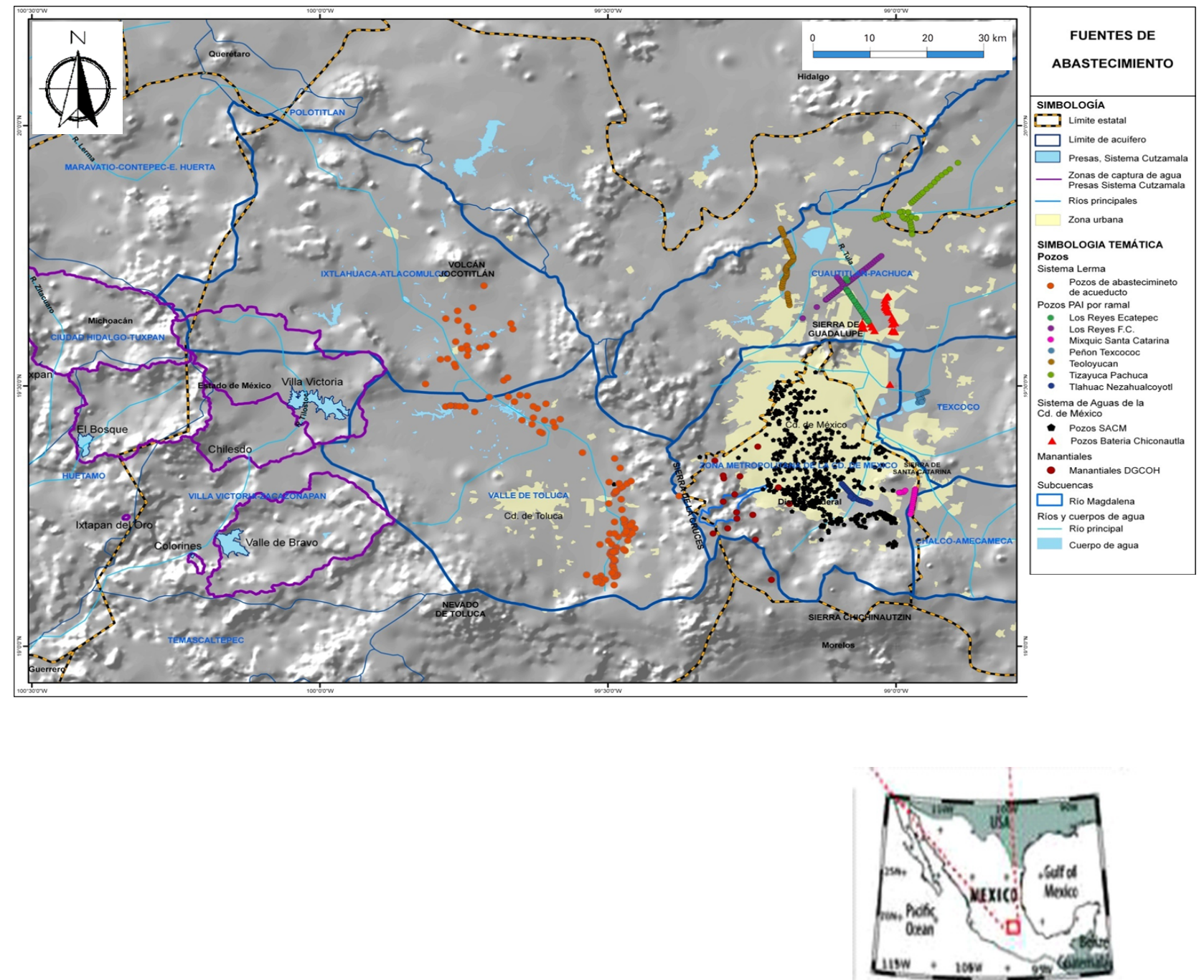

Figura 1 Localización de fuentes de abastecimiento de agua potable a la CM (modificado a partir de Escolero et al., 2009).

del Alto Río Lerma. La infraestructura cumple la función de captar y conducir el agua, y determina la capacidad del volumen de entrega; la componen presas, pozos, líneas de conducción, plantas de bombeo, etc.

\subsection{ANÁLISIS DE LOS FACTORES QUE INFLUYEN EN LA VULNERABILIDAD DE LAS FUENTES DE ABASTEGIMIENTO DE AGUA POTABLE}

Con base en una revisión bibliográfica exhaustiva se realizó la recopilación y revisión de bibliografía relacionada con la vulnerabilidad de los recursos hídricos y en particular en las fuentes de abastecimiento de agua potable, en el contexto internacio- nal, nacional y local, la revisión de metodologías para la determinación de la vulnerabilidad (cualitativa y cuantitativa), y la revisión de indicadores existentes a nivel internacional, nacional y local para la temática agua. Se llevó a cabo la revisión de indicadores internacionales y se comparó con los indicadores disponibles para México. Como fuentes internacionales se consideró la OCDE por su amplia difusión, el Departamento de Estadística de la ONU por su banco de indicadores a nivel mundial, los Sistemas de Indicadores de Estados Unidos y Canadá por su representación en la región de América del Norte, el PNUD por sus indicadores reconocidos internacionalmente (PNUD, 
2007), así como los utilizados por la Comisión Económica para Europa de las Naciones Unidas (UNECE) por su elaboración metodológica. La lista de indicadores de México se construyó a partir de diferentes documentos de la Secretaria de Medio Ambiente y Recursos Naturales (SEMARNAT, 2008).

En el marco de este trabajo, se llevó a cabo un taller de expertos con el fin de conocer y evaluar los factores que inciden en la vulnerabilidad de las fuentes de abastecimiento a la CDMX desde la perspectiva práctica-operativa, tomando como base los diagnósticos mencionados anteriormente. Los participantes fueron profesionales que participaron en el diseño, construcción, operación y gestión de las fuentes de abastecimiento a la GDMX. A partir de la información obtenida durante la primera parte de la discusión, se definió una lista de indicadores representativos. Para ello se agruparon los factores y problemas mencionados según se tratara de "infraestructura", "área de captación, y socio-administrativos, y posteriormente se determinaron aquellos indicadores que mejor resumían lo expuesto.

Posteriormente se analizan los resultados presentados en Martínez et al. (2015) a fin de establecer cómo estos factores o atributos inciden en la vulnerabilidad a fin de identificar áreas de oportunidad para reducir o mitigar dicha vulnerabilidad.

\section{Resultados}

\subsection{DIAGNÓSTICO DE LAS FUENTES DE ABASTECIMIENTO DE AGUA POTABLE}

\subsubsection{SISTEMA CUTZAMALA}

El Sistema Cutzamala aprovecha el agua de la cuenca alta del río del mismo nombre. Está conformado por las presas Tuxpan y el Bosque, en Michoacán; Colorines, Ixtapan del Oro, Valle de Bravo, Villa Victoria y Chilesdo, en el Estado de México. Con excepción de esta última, que se construyó para aprovechar el agua del río Malacatepec, los otros embalses formaban parte del Siste- ma Hidroeléctrico Miguel Alemán (Escolero et al., 2009 y Conagua, 2012b).

El sistema fue diseñado, construido y operado por el Gobierno Federal, y consistió de tres etapas, iniciando en 1982, con el aprovechamiento de la Presa Villa Victoria que aportó $4 \mathrm{~m}^{3} / \mathrm{s}$. La segunda etapa se concluyó en 1985 e incluyó el aprovechamiento de la presa Valle de Bravo la que aportó un caudal de $6 \mathrm{~m}^{3} / \mathrm{s}$ al sistema (Conagua 2012b). La tercera etapa se puso en funcionamiento en 1993 e integró los subsistemas Chilesdo y Colorines para sumar un aprovechamiento de 9 $\mathrm{m}^{3} / \mathrm{s}$. Además, lo integran seis macro plantas de bombeo que en conjunto vencen un desnivel de más de 1100 metros, un acueducto de $205.7 \mathrm{~km}$ con tubería de acero y concreto con diámetros entre 1.07 y 3.50 metros, $43.99 \mathrm{~km}$ de túnel, 72.55 $\mathrm{km}$ de canal abierto, y la planta potabilizadora Los Berros que consta de 5 módulos de 4000 L/s cada uno (CONAGUA, 2007a).

Las tres etapas del sistema originalmente fueron diseñadas para importar al Valle de México con un caudal máximo de 19 m³/s (599 Mm3/año), sin embargo, el sistema se ha operado normalmente con $16 \mathrm{~m} 3 / \mathrm{s}$ (505 $\mathrm{Mm}^{3} /$ año). Los números para el volumen de suministro actual varían, el SACMEX reporta una entrega de $6.73 \mathrm{~m}^{3} / \mathrm{s}$ (1993 - 2007) a la CDMX, el OCAVM reporta un volumen de $9.6 \mathrm{~m}^{3} / \mathrm{s}$. (Estos volúmenes podrían indicar la diferencia entre extracción y entrega, reflejando las fugas en la conducción). El volumen entregado al Estado de México es de alrededor de $6 \mathrm{~m}^{3}$ /s (CNA/IDECA, 1999).

$\mathrm{El}$ requerimiento anual de energía necesario para operar el sistema es de aproximadamente 1787 millones de $\mathrm{kWh}$. La energía consumida para bombear el volumen total de agua desde el sistema Cutzamala sólo hasta la planta de tratamiento equivale a la energía que consume la ciudad de Puebla, con una población de 8.3 millones de habitantes (Legorreta et al., 1997). El costo de operación se incrementa con el costo del personal y del proceso de tratamiento de agua, sin embargo, estos rubros representan sólo el $21 \%$ del costo total de operación. 
En la Tabla 2 se presenta un resumen de los principales factores que afectan la disponibilidad de agua en términos de cantidad y calidad del agua entregada por la fuente, tomando en cuenta los trabajos de Carnero-Bravo et al., 2014 y Figueroa-Sánchez et al., 2014; y en la Figura 2 se presenta el esquema y la problemática del sistema Cutzamala.

\subsubsection{SISTEMA LERMA}

Antes de la construcción del Sistema Cutzamala, la importación de agua desde la Cuenca del Alto Lerma hacia el Valle de México fue la única fuente externa de agua. Conforme los acuíferos presentaron fuertes impactos por la explotación intensiva, ésta fuente fue parcialmente sustituida por el Sistema Cutzamala.

La primera etapa del Sistema Lerma se llevó a cabo entre 1942 y 1951 e incluyó la captación de manantiales y agua superficial de Almoloya del Río y su conducción hasta los tanques de Dolores en Chapultepec, atravesando el túnel Atarasquillo - Dos Ríos (CONAGUA, 2007a). Originalmente se captó un caudal de $4 \mathrm{~m}^{3} / \mathrm{s}$ de la región Lerma situada aproximadamente a 300 metros por encima de la cota en que se encuentra la CDMX y se perforaron los primeros 5 pozos de entre $50 \mathrm{y}$ 308 metros de profundidad. Con la crisis de abastecimiento de agua a la CDMX. en la década de 1960, se firmaron convenios con el Gobierno del Estado de México (1965 - 1970) que terminaron en la construcción de una amplia batería de pozos en la cuenca del Alto Lerma (acuíferos Valle de Toluca e Ixtlahuaca-Atlacomulco) (CONAGUA, 2007a). Para agosto de 1970 se habían perforado 188 pozos de los cuales se extraían $10 \mathrm{~m}^{3} / \mathrm{s}$. Adicionalmente se construyeron los ramales de la Presa Alzate a Ixtlahuaca, el de Jiquipilco y los pozos de La Gavia. El proyecto incluyó un sistema de compensaciones al Estado de México, que consistió en la desecación de las lagunas locales para repartir 7000 ha de tierras agrícolas, el abastecimiento local para agua potable y agricultura, y la dotación de $1 \mathrm{~m}^{3} / \mathrm{s}$ de agua para los municipios de la zona conocida como región NZT (municipios de Naucalpan, Zaragoza y Tlalnepantla). No se cuenta con datos sobre el costo o consumo de energía para operar el acueducto y los pozos. Breceda-Lapeyre (2004) estimó el consumo de energía en $0.52 \mathrm{kWh} / \mathrm{m}^{3}$ para el sistema Lerma. En la Tabla 3 se presenta el resumen del diagnóstico del Sistema Lerma.

\subsubsection{SISTEMA DE POZOS PLAN DE ACCIÓN INMEDIATA (PAI)}

El Plan de Acción Inmediata (PAI) se elaboró en 1970 por la entonces Comisión de Aguas del Valle de México (CAVM) para satisfacer la creciente demanda de agua potable del Valle de México. La primera etapa del plan contempló el aprovechamiento transitorio de los acuíferos del Valle de México por medio de 9 baterías de pozos, así como la captación de agua superficial en la cuenca a través de la presa Guadalupe en el norte, y de 3 presas en la parte alta del Río Pánuco (CONAGUA, 2007b).

Tabla 2. Resumen del diagnóstico del sistema Cutzamala.

\begin{tabular}{|l|l}
$\begin{array}{l}\text { Factores que afectan el volumen } \\
\text { producido por las fuentes }\end{array}$ & $\begin{array}{l}\text { Problemas que implican real o } \\
\text { potencialmente un conflicto entre sus } \\
\text { usuarios }\end{array}$ \\
\hline
\end{tabular}

a) Variaciones en la precipitación y almacenamiento de las presas,

b) Problemas de funcionamiento en uno o más componentes del sistema,

c) Errores en la medición,

d) Extracciones clandestinas desde las conducciones,

e) Cambios en la demanda. a) Uso del agua en piscifactorías,

b) Venta de fuentes de abastecimiento (principalmente manantiales),

c) Escasez de agua para una parte de la población local,

d) Inundaciones y afectaciones de tierra vinculadas con el Sistema Cutzamala,

e) Contaminación del agua y

f) Extracción de agua del subsuelo.
Principales fuentes de contaminación que afectan a la Presa Valle de Bravo

a) descargas de agua residual,

b) desechos orgánicos y nutrientes provenientes de la acuacultura,

c) agua de retorno agrícola,

d) desechos sólidos y

e) erosión del suelo. 


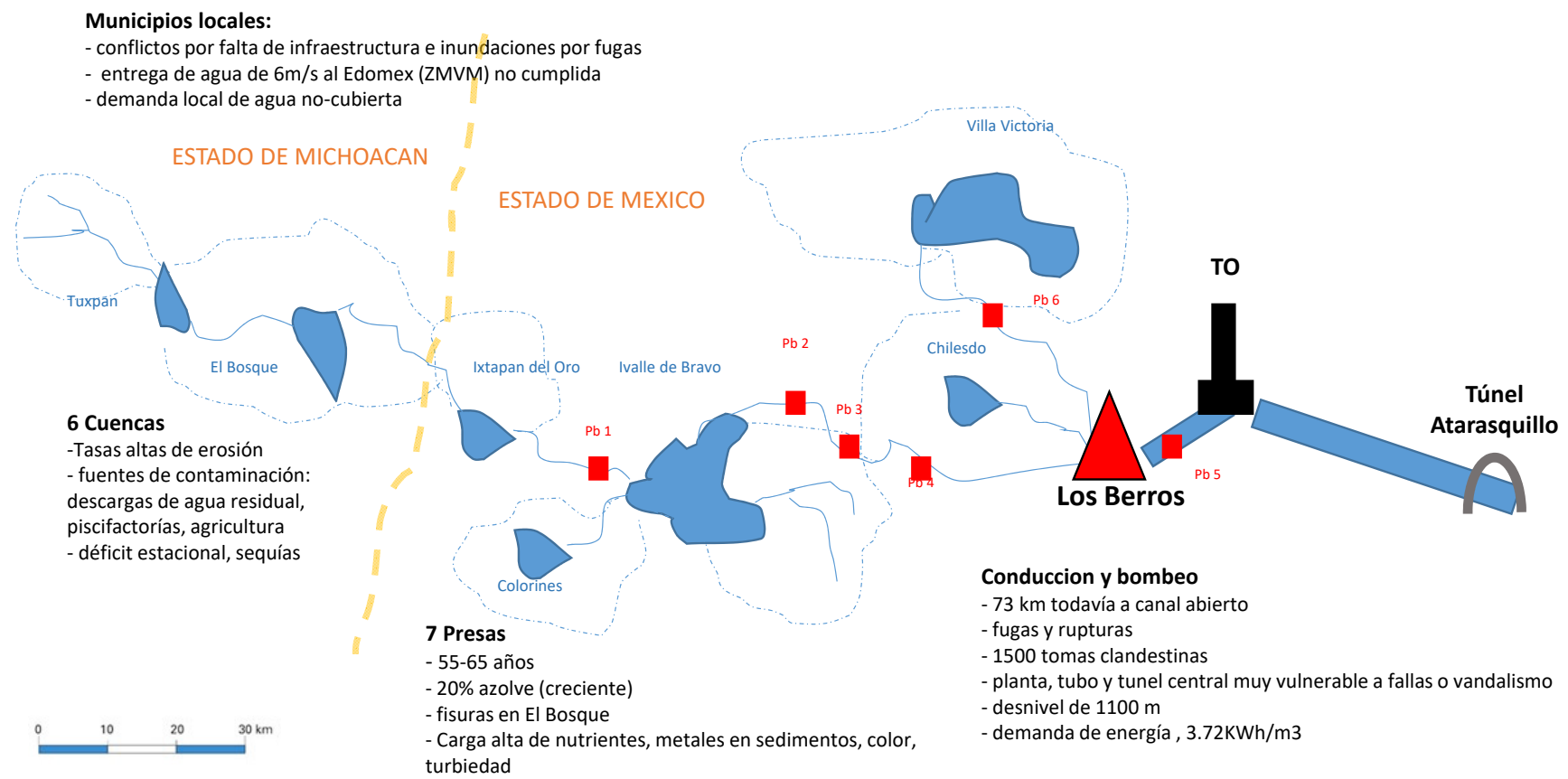

Los pozos del sistema PAI entraron en operación en 1974 como una solución temporal al problema de abasto y se convirtieron en una fuente regular y vital de suministro de agua en bloque para la CDMX y el Estado de México. De los pozos originales, en 1995 se transfirieron 84 al SACMEX y 70 pozos al Estado de México, y en 1997 fueron transferidos 17 pozos al Estado de Hidalgo (CONAGUA, 2007b).

El Sistema PAI es operado directamente por el OCAVM de la CONAGUA y en su totalidad abastece a 4 delegaciones de la CDMX, 2 municipios del Estado de Hidalgo y 14 municipios del Estado de México. En la Figura 3 se presenta la localización de los ramales del Sistema PAI.

Como ejemplo de los posibles conflictos originados por la demanda de agua en el origen de la fuente y que puede afectar la cantidad de agua entregada por la misma, se puede mencionar el municipio de Ecatepec en el Estado de México, donde se ubican 200 pozos para extracción de agua, de los cuales 50 pertenecen al Sistema PAI (Ramal Los Reyes-Ecatepec) operado por el OCAVM y el SACMEX (Sistema Chiconautla) para llevar agua a otras áreas de la ZMVM. En éste caso, el munici- pio de Ecatepec reclama que se le entreguen estos 50 pozos para compensar el déficit en el abastecimiento de agua potable que tiene el propio municipio.

\subsubsection{POZOS DEL SACMEX}

Los primeros pozos se perforaron en 1935 y la batería Milpa Alta en el sur fue la última en construirse en los años sesenta. Todos los pozos que opera el SACMEX se ubican dentro de la CDMX, con excepción de la Batería Chiconautla, la cual se tratará en otro apartado. Las baterías de pozos se integran en 5 sistemas según la región: Norte, Centro, Oriente, Sur, Poniente. Dentro de éstos, el Sistema Sur (el más grande con 284 pozos) se divide en los subsistemas Coyoacán, Tulyehualco, Pozos Aislados G.A.V.M, Tlalpan, Xochimilco, Milpa Alta y Auxiliar Xotepingo, mientras el Oriente con 94 pozos comprende las baterías Sistema Oriente, Ampliación Tláhuac, Agrícola Oriental y Santa Cruz Meyehualco. La profundidad promedio de los pozos es de $200 \mathrm{~m}$, con valores extremos de más de $1000 \mathrm{~m}$.

La extracción de agua del acuífero ZMVM por parte del SACMEX entre 1990 - 2008 ha sido de 
Tabla 3. Resumen del diagnóstico del sistema Lerma.

Factores que afectan el volumen
producido por los pozos

a) Producción e arena en los pozos,

b) Pozos suspendidos para no afectar la estabilidad de la Presa Alzate,

c) Pozos suspendidos por mala calidad del agua,

d) Agrietamientos y hundimientos del terreno por la extracción intensiva del agua

e) Pozos muy deteriorados debido a su antigüedad en operación.
Problemas que implican real o potencialmente un conflicto entre sus usuarios.

a) Demanda local de agua para riego agrícola,

b) Demanda local para abastecimiento de agua potable,

Factores de afectan la calidad del agua

a) Niveles del agua subterránea relativamente someros,

b) Numerosas fuentes potenciales de contaminación en superficie,

c) Demanda de agua potable de la Ciudad de c) Numerosos pozos abandonados, Toluca.

d) Infiltración de aguas residuales,

e) Descargas industriales al Río Lerma, e

f) Inversión de la dirección del flujo de agua subterránea.

Tabla 4. Características de los ramales del Sistema PAI.

\begin{tabular}{|l|c|c|c|c|c|c|}
\hline \multicolumn{1}{|c|}{ Ramal } & \# Pozos & En operación 2006 & Edad promedio & $\begin{array}{c}\text { Distancia mín. } \\
\text { entre pozos }\end{array}$ & $\begin{array}{c}\text { Problemas de } \\
\text { Calidad }\end{array}$ & $\begin{array}{c}\text { Abatimiento anual } \\
\text { del acuífero }\end{array}$ \\
\hline Tizayuca-Pachuca & 33 & 29 & 27 años & $360 \mathrm{~m}$ & $\left(\mathrm{HCO}_{3}\right)$ & $1-2 \mathrm{~m}$ \\
\hline Teoloyucan & 48 & 34 & 14 años & $220 \mathrm{~m}$ & $\mathrm{Na}, \mathrm{Cl}$ & $1-2 \mathrm{~m}$ \\
\hline Los Reyes-Ferrocarril & 49 & 38 & 23 años & $<200 \mathrm{~m}$ & $\mathrm{Na}, \mathrm{Cl}$ & $1-2 \mathrm{~m}$ \\
\hline Los Reyes-Ecatepec & 35 & 21 & 23 años & $200 \mathrm{~m}$ & $\mathrm{Na}, \mathrm{Cl}, \mathrm{SDT}$ & $2 \mathrm{~m}$ \\
\hline Tláhuac-Nezahualcoyotl & 20 & 14 & 17 años & $<150 \mathrm{~m}$ & $\mathrm{Mn}$ & $1 \mathrm{~m}$ \\
\hline Mixquic-S. Catarina & 19 & 9 & 21 años & $>300 \mathrm{~m}$ & $\mathrm{NH},(\mathrm{Na}, \mathrm{Cl}, \mathrm{Fe})$
\end{tabular}

Fuente: Elaboración propia con datos de CONAGUA, 2007b.

alrededor de $15 \mathrm{~m}^{3} / \mathrm{s}$, con un máximo en 2000 de $15.82 \mathrm{~m}^{3} / \mathrm{s}$ y una extracción mínima en 2009 de $13.93 \mathrm{~m}^{3} / \mathrm{s}$ (440 $\mathrm{Mm}^{3} /$ año).

\subsubsection{SISTEMA CHICONAUTLA}

El sistema Chiconautla se refiere a una batería de pozos en el Estado de México (Municipio de Ecatepec de Morelos) que capta agua del acuífero Cuautitlán-Pachuca. El sistema empezó a operar en 1957 para abastecer a la zona norte de la CDMX, según un acuerdo entre este municipio de Ecatepec y el Gobierno de la CDMX que incluyó el intercambio de agua subterránea por agua residual para el riego de cultivos. Incluía 3 ramales con un total de 39 pozos, además de 2 plantas de bombeo y tanques de almacenamiento con una capacidad de $105000 \mathrm{~m}^{3}$. Actualmente el sistema abarca 41 pozos con una profundidad entre $50 \mathrm{y}$ $321 \mathrm{~m}$. El agua se distribuye en el noreste de la CDMX. Del gasto inicial de $3.4 \mathrm{~m}^{3} / \mathrm{s}$, la extracción del sistema ha disminuido a casi la mitad. Las posibles causas de la reducción del caudal y las reposiciones son la edad de los pozos, el desgaste del equipo, el abatimiento del nivel del agua subterránea y problemas de calidad del agua. La profundidad al nivel estático del agua subterránea medida entre 2003 y 2005 por el SACMEX en 4 pozos fue de entre $52-67 \mathrm{~m}$, y el abatimiento se reporta entre 1.7 - 1.9 m/año. CONAGUA, en cambio reporta abatimientos anuales de hasta $2 \mathrm{~m}$ /año en la zona de Ecatepec para esas mismas fechas. 


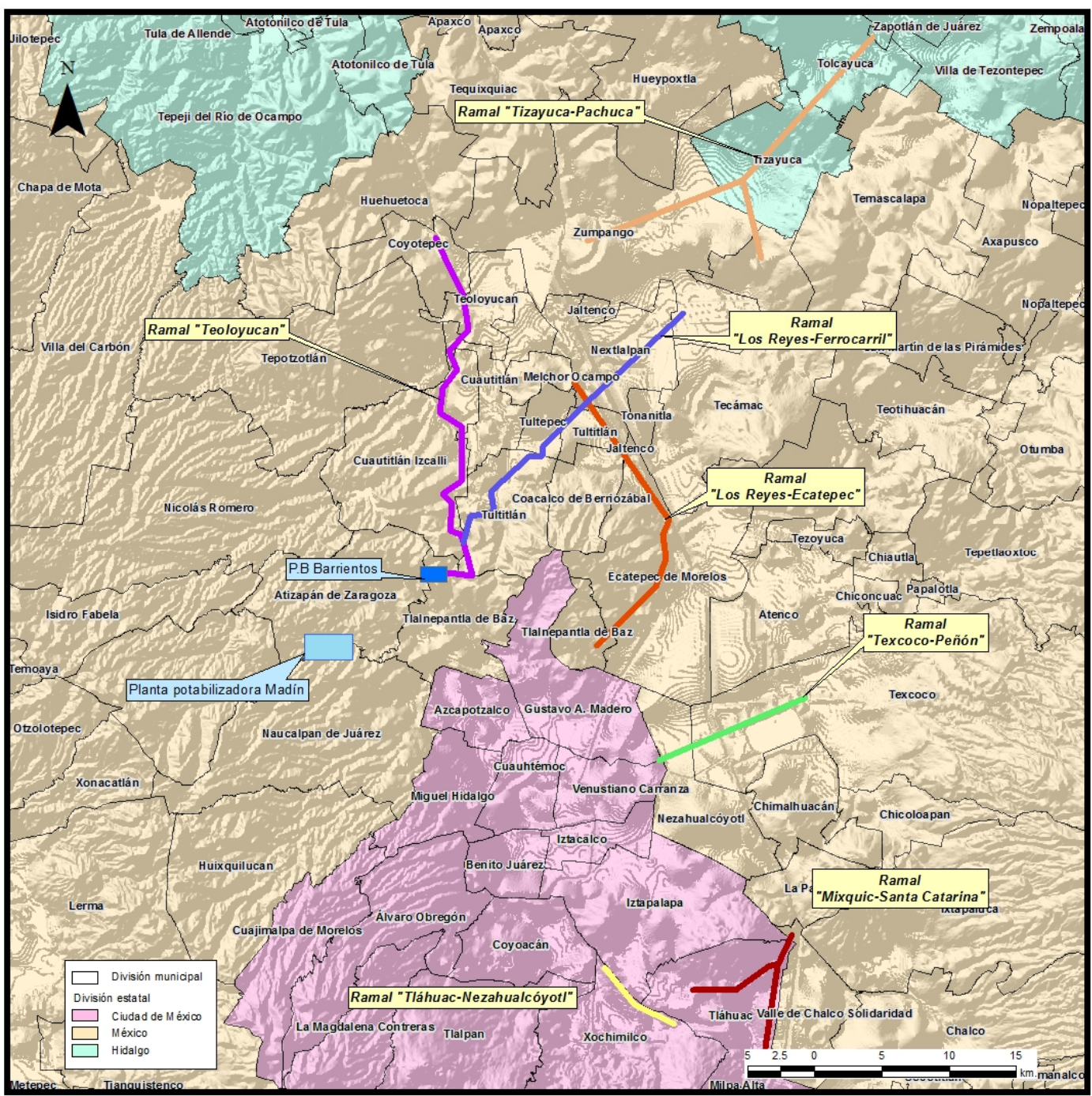

Figura 3 Localización de los ramales del Sistema PAl. Nota: Las líneas en colores representan las diferentes baterías o ramales a lo largo de las cuales están construidos los pozos (modificado a partir de Escolero et al., 2009).

\subsubsection{MANANTIALES}

El sistema de abastecimiento de la Ciudad de México incluye el aprovechamiento de 18 manantiales que en conjunto aportan $0.8 \mathrm{~m}^{3} / \mathrm{s}$. Se ubican en su totalidad en la parte oeste y suroeste de la CDMX dentro de la zona de suelos de conservación, a excepción de los manantiales Fuentes Brotantes, Peña Pobre y Santa Fe.

\subsection{ANÁLISIS DE LOS FAGTORES QUE INFLUYEN EN LA VULNERABILIDAD}

A partir de la información obtenida durante la etapa del taller de expertos, se definió una lista de indicadores representativos. Para ello se agruparon los factores y problemas mencionados por los invitados según se tratara de "infraestructura", "área de captación" o "situación socio-administrativa", y posteriormente se determinaron aquellos indicadores que mejor resumían lo expuesto. Los indicadores obtenidos se compararon con los resultados del diagnóstico, algunos de los cuales coincidieron, mientras que se agregaron otros. El resultado fueron 9 indicadores que representan la situación actual de las fuentes de abastecimiento y que permiten evaluar la vulnerabilidad a la infraestructura, vulnerabilidad ambiental y vulne- 
rabilidad socio-administrativa. Tres indicadores permiten evaluar cada índice de vulnerabilidad: a la infraestructura, ambiental y socio-administrativa. Los resultados se presentan en la Tabla 5 y se describen a continuación.

En relación a la infraestructura, el Sistema Cutzamala presenta el valor más elevado teniendo en cuenta el estado de las presas, los sistemas de conducción y la planta de tratamiento, así como las extracciones clandestinas que se registran anualmente. En general, en lo referente al "estado de la infraestructura" la falta de mantenimiento y adecuación de la infraestructura da valores altos para todos los sistemas de abastecimiento. Los pozos del Sistema PAI se encuentran más afectados debido a que la mayor parte están mal diseñados y construidos, provocando baja producción de agua, extracción de arena e interferencia durante el bombeo. El valor más bajo para este conjunto de indicadores fue asignado a la "exposición a daños por terceros" de los pozos SACM dado que cuentan con una adecuada infraestructura de protección y con vigilancia. En relación a la capacidad de extracción de los pozos del Sistema Lerma, la mayor parte opera por debajo de su capacidad de diseño pudiendo ser incrementada.

En relación a los aspectos ambientales cabe aclarar que para la "calidad" se tuvo en cuenta la calidad del agua en la fuente, mientras que el riesgo de contaminación en las áreas de captación (basureros, industrias, etc.) fue considerado en la "degradación ambiental". La mayor vulnerabilidad ambiental la presenta igualmente el Sistema Cutzamala dado la reducción de la capacidad de almacenamiento en las presas, los altos niveles de degradación hídrica en las cuencas de captación, la contaminación del agua en las presas y la fuerte presencia de fuentes contaminantes, entre los factores más importantes. En segundo término, los pozos de SACMEX muestran alta vulnerabilidad, debido a los fuertes descensos del nivel del agua, cambios en la calidad del agua extraída y altos niveles de degradación, debido a su localización dentro de la zona urbana, la presencia de fuentes contaminantes y hundimiento del suelo. La ausencia de información respecto a la calidad del agua de los sistemas Lerma y Chiconautla impide la valoración completa de la vulnerabilidad ambiental. Los factores disponibilidad y degradación en el sistema Chiconautla son tan elevados como para los pozos SACM. Para el Sistema Lerma la valoración con respecto a la disponibilidad está relacionada con los registros que muestran la relativa estabilización de los niveles del agua en los últimos años y reportes que indican la factibilidad de incrementar la extracción con descensos moderados adicionales en los niveles. En la valoración del sistema PAI se tomó en cuenta que los ramales del sur, los cuales presentan graves problemas de calidad del agua y descensos de niveles estáticos, aportan un volumen reducido en comparación a los ramales del norte con mejores condiciones de cantidad y calidad de agua aportada.

La vulnerabilidad socio-administrativa es asimismo alta para el Sistema Cutzamala teniendo en cuenta las demandas de grupos sociales localizados en las áreas de captación y la baja eficiencia económica del sistema. Dado que este sistema fue diseñado, construido y es gestionado únicamente por el Gobierno Federal, la situación desde el

Tabla 5. Evaluación de índices de vulnerabilidad de fuentes de agua potable.

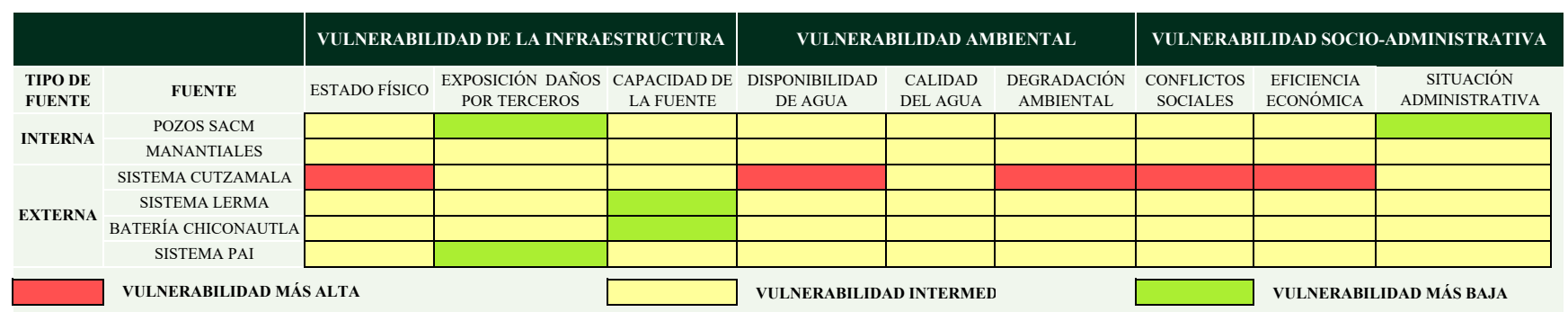

Elaborada con datos de Escolero et al. (2009) y Martínez et al. (2015). 
punto de vista administrativo no representa graves problemas aparentemente. En segundo lugar, la vulnerabilidad del Sistema Lerma está dada por los conflictos sociales y baja eficiencia económica, si bien con menor gravedad que en el Sistema Cutzamala. Sin embargo, la situación administrativa del Sistema Lerma es mayor que para Cutzamala debido a la participación de dos organismos en la gestión (CONAGUA y SACM) y al complejo sistema de compensación al Estado de México operado por el SACM. La menor vulnerabilidad socio-administrativa la presentan los pozos del SACM debido a que son gestionados y operados por la misma entidad, si bien existen algunos conflictos por demandas locales y una eficiencia que requiere ser incrementada.

Un resultado importante de la valoración es que las 3 dimensiones - infraestructura, estado ambiental del área de captación y las condiciones sociales-administrativas- tienen un impacto comparable en la vulnerabilidad de las fuentes de abastecimiento. Por lo tanto, cada uno de estos factores debe ser parte equitativa en las soluciones para mejorar la situación actual y la capacidad de adaptación a futuros cambios.

\section{Discusión}

La intervención de dependencias federales, estatales y municipales en la gestión del agua en la zona conurbada de la CDMX, ha contribuido a la falta de planeación que ha llevado a la explotación intensiva del recurso y al déficit regional. $\mathrm{Al}$ mismo tiempo, la práctica del "tributarismo" hídrico para satisfacer la demanda urbana está generando e incrementando conflictos sociales y políticos, por lo que estos factores incrementan la vulnerabilidad de las fuentes de abastecimiento de agua. Esto se refleja en que los consumos mínimos de agua en la CDMX se registran en algunos asentamientos ilegales con alrededor de $28 \mathrm{~L} / \mathrm{hab} /$ día, mientras que la estimación para las zonas de sectores medios es entre 275 y $410 \mathrm{~L} / \mathrm{hab} /$ día y en los sectores de máximos ingresos entre 800 y $1000 \mathrm{~L} / \mathrm{hab}$ /día
(Legorreta et al., 1997). Sin embargo, éstos consumos deben tomarse con reservas debido a que toman en cuenta valores de macro medición y no consumos reales.

Por otra parte, el hundimiento del terreno está considerado como el problema ambiental más importante de la ciudad, y se atribuye al drenado de las arcillas que sobreyacen al acuífero por efecto de la extracción y las fuerzas verticales de las construcciones. El hundimiento varía entre 6 y 30 centímetros al año (Chaussard et al., 2014). Una tercera parte de este problema se atribuye a la impermeabilización por la urbanización y dos terceras partes a la extracción de agua subterránea (Sheinbaum, 2004). El hundimiento diferencial causa daños a la infraestructura urbana, y se considera responsable del alto índice de fugas en los sistemas de agua y drenaje $(32-38$ \% según diferentes fuentes). El fenómeno tiene otra manifestación en los contactos abruptos entre la zona lacustre y los aparatos volcánicos (p. ej. alrededor de la Sierra Santa Catarina, Guadalupe, y Cerro de la Estrella), en donde se observa la formación de fisuras superficiales con aberturas considerables que dañan la infraestructura y ponen en riesgo a la población (Andrade et al., 2013). Se estimaba que sólo los materiales localizados a profundidades menores a $80 \mathrm{~m}$ eran compresibles debido al alto contenido de arcillas, sin embargo, mediciones de 2004 indican una alta compresibilidad también en la formación de limos arenosos (entre $80-160 \mathrm{~m}$ de profundidad), al menos en el centro de la ciudad (Santoyo et al., 2005). Por lo tanto, el fenómeno de hundimiento podría afectar al sistema de drenaje profundo, reduciendo su eficiencia hidráulica y dañando los túneles.

Si bien el sistema de abastecimiento por medio de pozos en la CDMX no tiene los problemas inherentes a la importación de agua de otros estados, existen fuertes desigualdades y conflictos potenciales por transferencias de agua entre diferentes municipios y delegaciones (Wigle, 2010; Starkl et al., 2013; Banister y Widdifield, 2014). En general se puede decir que la calidad y cantidad del abastecimiento es fundamentalmente desigual, tendiendo 
a decrecer hacia el oriente de la ciudad donde se ubican las delegaciones con mayor crecimiento y menor acceso a las fuentes externas de agua. Casi todas las delegaciones tienen coberturas de agua potable por arriba de $90 \%$, con excepción de Xochimilco, Milpa Alta y Tlahuac. Sin embargo, esto no dice nada sobre la calidad del servicio otorgado, tanto en cantidad (tan solo en Iztapalapa más de 90 colonias reciben el agua por tandeo) como en calidad.

La delegación Xochimilco es un buen ejemplo de trasvases de agua dentro de la CDMX a costa de la sustentabilidad y la demanda insatisfecha local. Tradicionalmente ha sido la zona proveedora de agua a la CDMX y la desecación de sus manantiales ha permitido el crecimiento de otras delegaciones mucho antes de que Xochimilco se incorporara a la zona conurbada de la CDMX. Actualmente, se extraen alrededor de $0.7 \mathrm{~m}^{3} / \mathrm{s}$ por el sistema PAI y $2.6 \mathrm{~m}^{3}$ /s por el SACM. Sin embargo, la Delegación Xochimilco no recibe agua al menos de la primera fuente y muchas comunidades de la delegación se abastecen con agua de la Delegación Milpa Alta. La extracción intensiva está amenazando al lago, los canales y manantiales, y causa hundimientos del terreno.

Es importante considerar que las áreas de conservación cumplen un rol fundamental ya que están ligadas a las zonas de recarga del acuífero y los manantiales que abastecen a la CDMX. Un manejo adecuado de estas áreas es prioritario para la conservación de la cantidad y calidad del recurso hídrico. En los últimos años, estas áreas han estado en un riesgo creciente debido al crecimiento urbano. Entre 1980 - 2000, buena parte de las 377000 nuevas casas que fueron construidas en la CDMX se localizaron en las áreas de conservación. De los 44 asentamientos rurales que todavía existen en la CDMX, 35 de ellos (400000 personas) están situados en áreas de conservación (Tortajada, 2006). Adicionalmente, la expansión de asentamientos ilegales se ha convertido en un problema crítico. En 2003 se reportaron 804 asentamientos irregulares con aproximadamente 60000 familias, $80 \%$ de las cuales fueron en áreas de conservación.
Si bien los indicadores determinados para México coinciden en buena parte con indicadores internacionales, estos representan solo una parte de la problemática del abastecimiento de agua. Los indicadores disponibles para México representan la accesibilidad al servicio de agua potable y drenaje (medida a escala municipal), la disponibilidad de agua en relación a la cantidad de población, la calidad de los cuerpos de agua y el nivel de tratamiento de aguas residuales, entre otros. Sin embargo, éstos no representan las condiciones relevantes a la escala de las fuentes de abastecimiento con excepción de unos pocos, tal como el contenido de nitrato y fósforo en aguas superficiales o capacidad de almacenamiento en presas. Estos indicadores, como otros, tienen la limitante de ser aplicables sólo a fuentes superficiales como el Sistema Cutzamala. Adicionalmente, datos químicos y otros de relevante importancia, no están disponibles para las escalas temporal y espacial requeridas para el presente análisis. La falta de datos explica el carácter no prioritario que tiene el desarrollo de un sistema de indicadores a nivel regional. Según datos de la Secretaria del Medio Ambiente, los recursos destinados al ramo ambiental representan el $0.23 \%$ del gasto del Gobierno Federal; más aún, sólo el $0.2 \%$ de esa cantidad está destinado al Sistema de Indicadores de Sustentabilidad.

La valoración obtenida en el taller de expertos se destaca en general por la percepción de los efectos en lugar de las causas. Por ejemplo, mientras se consideran de alta importancia el azolve en las presas y el deterioro de la calidad del agua, se asignaron valores bajos al factor de degradación en las cuencas. De igual manera, las extracciones clandestinas y el riesgo al vandalismo se juzgaron más importantes que su causa, que es en gran medida la demanda local de agua. Asimismo, se observa que los factores con los cuales los asistentes no han estado directamente involucrados, son considerados como daños colaterales en lugar de causa de los problemas. Esto explica la falta de acuerdos para la resolución de conflictos y atención a las demandas locales, y la persistencia al enfoque de incrementar el abastecimiento con fuentes le- 
janas. En relación al cambio climático se reconoce su importancia para la disponibilidad de agua en fuentes superficiales (Martínez et al., 2015), sin embargo, el alto consumo de energía que requiere la importación de agua sigue siendo un costo que la mayoría de los asistentes justifica y el cual lo relacionan con un costo monetario y no con su impacto sobre el clima.

La vulnerabilidad total obtenida muestra congruencia con el diagnóstico y los resultados del taller. Actualmente, el sistema de abastecimiento más vulnerable es Cutzamala, mientras que el sistema menos vulnerable corresponde a los pozos del SACM en la zona urbana de la CDMX. En la alta vulnerabilidad del Sistema Cutzamala influye: i) la reducción en la disponibilidad de agua teniendo en cuenta que los sistemas superficiales responden rápidamente a los efectos de la alta degradación en las áreas de captación y variaciones de los parámetros climáticos (Mazari et al., 2014); ii) la falta de inversión en mantenimiento y rehabilitación de la infraestructura por largos períodos; iii) los conflictos por demandas sociales locales que genera este sistema de abastecimiento; y iv) los elevados costos de operación en relación al volumen aportado. La menor vulnerabilidad del abastecimiento por pozos del SACM está influenciada por: i) la baja exposición a daños por terceros; ii) la gestión a cargo de un único organismo que cuenta con los derechos para la extracción de agua; y iii) una relación costo-beneficio y capacidad de extracción media.

En este sentido, recientemente el SACM ha iniciado un programa de exploraciones profundas, con la perforación de un pozo a 2008 metros de profundidad en el sitio denominado San Lorenzo Tezonco, con resultados satisfactorios (Arce et al., 2013 y Morales-Casique et al., 2014) con lo que se espera extraer agua subterránea de un estrato acuífero localizado a más de 1000 metros de profundidad. Ante este resultado, ya se han programado otras perforaciones profundas dentro de la CDMX, con las cuales se podrán responder cuestionamientos sobre la posible conexión hidráulica con los estratos acuíferos superiores.

\section{Conclusiones}

Durante el diagnóstico se ha visto que la infraestructura y los factores socio-administrativos juegan un rol tan importante en la vulnerabilidad de las fuentes de abastecimiento, como los factores ambientales. Es evidente, que los sistemas de abastecimiento operan al límite y no llegan a satisfacer la demanda de agua que presenta la Zona Metropolitana del Valle de México. A la situación de alta vulnerabilidad actual se debe agregar los posibles efectos del cambio climático, así como los cambios por: i) crecimiento en la demanda de agua; ii) aumento de la degradación en las áreas de captación; iii) reducción de la calidad de agua y recarga, entre otros. Para garantizar el abastecimiento a futuro, será necesario desarrollar nuevas fuentes de agua potable. El hecho de que el Sistema Cutzamala como fuente externa de agua superficial presenta la vulnerabilidad más alta, apunta a la necesidad de un cambio de paradigma en la planeación de futuras fuentes. Los mismos procesos climáticos, sociales y ambientales que están disminuyendo la disponibilidad de agua entregada por esta fuente, podrían afectar los demás proyectos de importación de otras cuencas y aún a mayor costo. La alta vulnerabilidad por la degradación ambiental que presentan las fuentes de abastecimiento de origen superficial obliga a reevaluar los proyectos de importación de agua desde el punto de vista de la disponibilidad y la degradación ambiental. Los cambios en la disponibilidad de agua como posible efecto del cambio climático en la región, han sido desestimados. Estas variaciones no han sido tomadas en cuenta en los proyectos para una futura importación de agua superficial, por lo que requieren una reevaluación.

Mientras el nuevo paradigma de gestión de agua urbana, a nivel internacional, promueve el aprovechamiento integral y descentralizado de los recursos de agua disponible, en la Ciudad de México aún prevalecen los enfoques tradicionales de gestión centralizada y aprovechamiento de agua de 
primer uso. La falta de planeación de los sistemas de agua obliga a medidas de emergencia que impactan en los costos y la sustentabilidad de los sistemas de agua urbana. Un ejemplo es la descarga y disposición conjunta de agua pluvial y residual que inhibe el uso descentralizado e incrementa los costos de inversión y tratamiento, entre otros efectos negativos.

Igual que la importación de agua, el uso del agua residual para potabilización presentará objeciones sociales, con la única diferencia que éstas se presentarían por los propios electores, lo cual aumenta el grado de dificultad para su aplicación en la práctica. Sin embargo, con voluntad política y convenientes campañas de información, el uso del agua tratada para agua potable puede constituir una solución viable que fomente una cultura de agua orientada en las realidades y necesidades locales, en lugar de conceptos abstractos.

A su vez, los esfuerzos de separar las aguas pluviales de las residuales se han visto impedidos por la falta de opciones superficiales para almacenar el agua de lluvia dentro de la cuenca. Sin embargo, gracias a los avances en la tecnología de manejo de recarga de acuíferos, es posible utilizar estos acuíferos como almacenamientos gigantescos, con lo que los problemas causados por inundaciones y costo de exportación podrían ser superados. Sin embargo, el problema del tratamiento de agua y ubicación adecuada de las obras es complejo. No obstante, se han hecho avances tanto por el SACM como por parte del Gobierno Federal. Aquí sería importante que se junten los esfuerzos de la CONAGUA y del SACM, (así como de las instituciones académicas). No es aceptable que una solución que está a la mano y cuya aplicación es urgente, no se aproveche al máximo por problemas administrativos, políticos o falta de coordinación.

La protección de las partes altas de las cuencas debe ser considerada como uno de los temas prioritarios para la protección ambiental y civil, el cuidado de las zonas de recarga y la conservación del clima local. Esto requiere la declaración de las zonas de recarga como Áreas Naturales Protegidas (adicionales a las ya existentes) pero sobre todo la implementación práctica de estos decretos. Es contradictoria una política en la que se propone la reforestación intensa, pero sin el control necesario para proteger los recursos forestales existentes.

En el contexto del cambio climático y la demanda creciente, las soluciones deben ser buscadas en el manejo descentralizado del ciclo de agua urbana para encontrar los usos y demandas locales. Esto disminuye los costos de inversión y mantenimiento, reduce el consumo de energía y facilita el almacenamiento y la distribución.

Un análisis global de los impactos y beneficios que cada propuesta conlleva, así como de las opciones que el sistema actual presenta para incrementar la oferta, es necesario antes de tomar decisiones. Soluciones socialmente aceptables y ambientalmente deseables, deberían considerar primero estrategias enfocadas a la demanda, antes de privilegiar los proyectos de importación de agua para aumentar la oferta.

\section{Agradecimientos}

La investigación presentada en este trabajo fue financiada por el Centro Virtual de Cambio Climático de la Ciudad de México (GVCGCM), como parte de los proyectos para el desarrollo de conocimientos, metodologías, estrategias y políticas de adaptación al cambio climático a través del esfuerzo integrado de los sectores público, privado, social y científico. Los autores desean agradecer a los gestores del agua y los expertos que participaron en el taller la contribución con su tiempo y sus conocimientos sobre los sistemas de abastecimiento de agua de la CDMX. Así mismo, desean agradecer a los revisores Dr. José Manuel Murillo y otros dos revisores anónimos, cuyas observaciones y sugerencias contribuyeron a mejorar mucho este trabajo. 


\section{Referencias}

Andrade, J., Martin, A., Rodríguez, A., 2013, Knitting networks: Transforming Chalco Channel, Mexico City. The Sustainable City: WIT Transactions on Ecology and The Environment, 179, 579-590.

Arce, J.L., Layer, P.W., Morales-Casique, E., Benowitz, J.A., Rangel, E., Escolero, O., 2013, New constraints on the subsurface geology of the Mexico City Basin: The San Lorenzo Tezonco deep well, on the basis of ${ }^{40} \mathrm{Ar} /{ }^{39} \mathrm{Ar}$ geochronology and whole-rock chemistry: Journal of Volcanology and Geothermal Research, 266, 34-49.

Banister, J.M., Widdifield, S.G., 2014, The debut of 'modern water' in early $20^{\text {th }}$ century Mexico City: The Xochimilco potable water works: Journal of Historical Geography, 46, 36-52.

Birkle, P., Torres-Rodriguez, V., GonzálezPartida, E., 1998, The water balance for the Basin of the Valley of Mexico and implications for future water consumption: Hydrogeology Journal, 6, 500-517.

Breceda-Lapeyre, M.C., 2004, Agua y energía en la Ciudad de México (Visión en 2004). Seminario Internacional del Agua ¿Bien privado o bien común?, septiembre 22, 2004. Universidad de la Giudad de México.

Carnero-Bravo V., Merino-Ibarra M., RuizFernández A.C., Sánchez-Cabeza J.A., Ghaleb, B., 2014, Sedimentary record of water column trophic conditions and sediment carbon fluxes in a tropical water reservoir (Valle de Bravo, Mexico): Environmental Science Pollution Research 22(6), 4680-4694.

Carrera-Hernández, J.J., Gaskin, S.J., 2009, Water management in the Basin of Mexico: current state and alternative scenarios: Hydrogeology Journal, 17(6), 1483-1494. Comisión Nacional del Agua, IDECA, Investigación y Desarrollo de Estudios de
Calidad del Agua (CNA/IDECA), 1999, Estudio Limnológico de la Presa Valle de Bravo, Informe Final, Contrato GT-306GAVM-98.

Comisión Nacional del Agua (CONAGUA), 2007a, Estadísticas del agua 2007 de la Región XIII. Organismo de Cuenca Aguas del Valle de México.

Comisión Nacional del Agua (CONAGUA), 2007b, Plan de Acción Inmediata.

Organismo de Cuenca Aguas del Valle de México.

Comisión Nacional del Agua (CONAGUA), 2009, Estadísticas de la Región Hidrológica Administrativa XIII, Aguas del Valle de México, Reporte Técnico de la Comisión Nacional del Agua, México.

Comisión Nacional del Agua (CONAGUA), 2012a, Estadísticas del Agua en México. Reporte Técnico de la Comisión Nacional del Agua, México.

Comisión Nacional del Agua (CONAGUA), 2012b, Programa Hídrico Regional Visión 2030. Región Hidrológico-Administrativa XIII Aguas del Valle de México, Reporte de la Comisión Nacional del Agua, México.

Comisión Nacional del Agua, Instituto Mexicano de Tecnología del Agua (CONAGUA/ IMTA), 2006, Diagnóstico de la cuenca Valle de Bravo. Plan para la gestión integral del agua y recursos asociados de la Cuenca de Valle de Bravo, Estado de México.

Convenio GAVMSG-GOA-MEX-05-404RF-CG.

Chaussard, E., Wdowinski, S., Cabral-Cano, E., Amelung F., 2014, Land Subsidence in Central Mexico detected by ALOS InSAR time-series: Remote Sensing of Environment, 140, 94-106.

Chia-Ling, C., Chung-Hsin, H., 2011, Applying a Modified VIKOR Method to Classify Land Subdivisions According to Watershed Vulnerability: Water Resources Management, 25, 301-309. 
Downing, T.E., Butterfield, R., Cohen, S., Huq, S., Moss, R., Rahman, A., Sokona, Y., Stephen, L., 2001, Climate Change Vulnerability: Linking Impacts and Adaptation. Report to the Governing Council of the United Nations Programme, United Nations Environmental Programme. Nairobi, Kenya.

Banco Mundial, El, 2013, Agua Urbana en el Valle de México: Un Camino Verde para Mañana. México City, disponible en <http://www-wds.worldbank.org/ external/default/WDSContentServer/ WDSP/IB/2013/03/12/0004259 62_20130312111818/Rendered/, 59170WP0P11990n0el0Valle0de0Mexico. pdf>, accesado en 15/06/2013.

Escolero, O., Martínez, S.E., Kralisch, S., Perevochtchikova, M. Delgado-Campo, J., 2009, Vulnerabilidad de las fuentes de abastecimiento de agua potable de la Ciudad de México en el contexto de cambio climático: Informe final. Centro Virtual de Cambio Climático.

Figueroa-Sánchez, M., Nandini, S., Sarma, S., 2014, Zooplankton community structure in the presence of low levels of cyanotoxins: a case study in a high altitude tropical reservoir (Valle de Bravo, Mexico): Journal of Limnology, 73(1), 157-166.

González-Reynosa, A., Jiménez-Cisneros, B., Gutiérrez-Rivas, R., 2010, Evaluación externa del diseño e implementación de la política de acceso al agua potable del gobierno del Distrito Federal. Reporte Técnico del Programa Universitario de Estudios sobre la Ciudad de la UNAM. Consejo de Evaluación del Desarrollo Social del Distrito Federal, México, México. Hamouda, M.A., Nour El-Din, M.M., Moursy, F.I., 2009, Vulnerability Assessment of Water Resources Systems in the Eastern Nile Basin: Water Resources Management 23, 2697-2725.
Kraas, F., 2008, Megacities as Global Risk Areas: Urban Ecology (J. Marzluff, E. Shulenberger, W. Endlicher et al. eds). Springer US, 583-596.

Legorreta, J., Contreras, M.C., Flores, M.A., Jiménez, N., 1997, Agua y más agua para la Ciudad, Red Mexicana de Eco-Turismo, disponible en <http://www.planeta.com/ ecotravel/mexico/ecologia/97/0897agua. html>, accesado en 28/07/2015.

Luers, A.L., Lobell, D.B, Sklar, L.S, Addams, C.L., Matson, P.A., 2003, A method for quantifying vulnerability, applied to the agricultural System of the Yaqui Valley, Mexico: Global Environmental Change 13, 255-267.

Martínez, S.E., Delgado, J., Escolero, O., et al., 2009, Socio-Economic Development in Arid Zones: The Influence of Water Availability in the San Luis Potosi Basin, Mexico, en Fernandez-Bernal, A., De La Rosa, M.A., (eds.), Arid environments and wind erosion, Nova Science Publishers, NY, EEUU, ISBN: 978-1-60692-411-2.

Martínez, S.E., Escolero, O., Wolf, L., 2011, Total Urban Water Cycle Models in Semiarid Environments - Quantitative Scenario Analysis at the Area of San Luis Potosi, Mexico: Water Resources Management 25, 239-263.

Martínez, S., Kralisch, S., Escolero, O., Perevochtchikova, M., 2015, Vulnerability of Mexico City`s water supply sources in the context of climate change: Journal of Water and Climate, 6(3), 518-533.

Mazari-Hiriart, M., Pérez-Ortiz, G., OrtaLedezma, M.T., Armas-Vargas, F., Tapia M.A., Solano-Ortiz, R., Silva, M.A., YañezNoguez, I., López-Visal, Y., Díaz-Ávalos, C., 2014, Final Opportunity to Rehabilitate an Urban River as a Water Source for Mexico City: Plos One, 9(7) Article number e102081. 
Mendoza, V., Villanueva, E., Maderey, L., 2008, Vulnerabilidad en el recurso agua de las zonas hidrológicas de México ante el cambio climático global: En Cambio climático: una visión desde México, en Martínez J., Fernández, A. (eds.), Secretaria de Medio Ambiente y Recursos Naturales, Instituto Nacional de Ecología, México City, 15, 227 p.

Mitchell, V.G., 2006, Applying integrated urban water management concepts: a review of Australian experience: Environmental Management 37(5), 589-605.

Morales-Casique, E., Escolero, O.A., Arce, J.L., 2014, Resultados del pozo San Lorenzo Tezonco y sus implicaciones en el entendimiento de la hidrogeología regional de la cuenca de México: Revista Mexicana de Ciencias Geológicas, 31(1), 64-75.

Morris, B.L., Lawrence, A.R.L., Chilton, P.J.G., Adams, B., Calow, R.C., Klinck, B.A., 2003, Groundwater and its Susceptibility to Degradation: A Global Assessment of the Problem and Options for Management. Early Warning and Assessment Report Series, RS. 03-3. United Nations Environment Programme, Nairobi, Kenya. ISBN: 92-807-2297-2, 126 p.

Moss, R.H., Malone, E.L., Brenkert, A.L., 2002, Vulnerability to climate change: a quantitative approach. Paper prepared for the US Department of Energy, disponible en <http://www.globalchange.umd.edu/data/ publications/Vulnerability_to_Climate_ Change.PDF>, accesado en 28/07/2015.

Olmos, S., 2001, Vulnerability and adaptation to climate change: Concepts, issues, assessment methods. Paper prepared for the Climate Change Knowledge Network, disponible en $<$ http://www.start.org/Projects/AIACG_ Project/meetings/Trieste_02/trieste_cd/ Resource_Materials/CGKN.pdf>, accesado en 28/07/2015.
Pandey, R.P., Pandey, A., Galkate, R.V., Byun, H.R., Mal, B.C., 2010, Integrating HydroMeteorological and Physiographic Factors for Assessment of Vulnerability to Drought: Water Resources Management, 24, 41994217.

Pinto, J., Varum, H., Bentes, I., Agarwal, J., 2010, A Theory of Vulnerability of Water Pipe Network (TVWPN): Water Resources Management 24, 4237-4254.

PNUD, 2007, Indicators of sustainable development: guidelines and methodologies. United Nations, ISBN 978-92-1-104577-2, $94 \mathrm{p}$.

Pritchett, L., Suryahadi, A., Sumarto, S., 2000, Quantifying vulnerability to poverty: A proposed measure with application to Indonesia: Social Monitoring and Early Response Unit Research Institute (SMERU). Working Paper, disponible en <http://www. smeru.or.id>.

Santoyo-Villa, E., Ovando-Shelley, E., Mooser, F., León-Plata, E., 2005, Síntesis Geotécnica de la Cuenca del Valle de México: Ciudad de México, TGC Ediciones, 171 p.

SEMARNAT, 2008, Indicadores básicos del desempeño ambiental de México, México, Edición 2008, disponible en <http://apps1.semarnat.gob.mx/dgeia/ indicadores_2008/, accesado en 28/07/ 2015>.

Sena, J.A., Freitas, M.A.V., Berrêdo, D., CostaFernández, L., 2012, Evaluation of Vulnerability to Extreme Climatic Events in the Brazilian Amazonia: Methodological Proposal to the Rio Acre Basin: Water Resources Management (2012) 26, 45534568.

Sheinbaum, C., 2004, Proyecto de recarga del acuífero en el sur del Distrito Federal. Conferencia en el Colegio de Ingenieros Civiles de México, febrero 18 de 2004: Revista Tláloc, Asociación Mexicana de Hidráulica, 30, enero-abril 2004, 43-44. 
Starkl, M., Brunner, N., Lopez, E., MartinezRuiz, J.L., 2013, A planning-oriented sustainability assesment framework for periurban water management in developing countries: Water Research, 47, 7175-7183.

Tortajada, C., 2006, Water management in Mexico City Metropolitan Area: International Journal of Water Resources Development, 22(2), 353-376.
Wigle, J., 2010, The 'Xochimilco model' for managing irregular settlements in conservation land in Mexico City: Cities, 27, 337-347.

Wilhelmi, O.V., Morss, R.E., 2013, Integrated analysis of societal vulnerability in an extreme precipitation event: a Fort Collins case study: Environmental Science \& Policy, 26, 49-62. 